\title{
Modelling skin penetration using the Laplace transform technique
}

\author{
Yuri G. Anissimov ${ }^{1 *}$, Adam Watkinson ${ }^{2}$
}

${ }^{1}$ Griffith University, School of Biomolecular and Physical Sciences and Queensland Micro- and Nanotechnology Centre, Gold Coast, QLD 4222, Australia.

${ }^{2}$ Storith Consulting Limited, 138 High Street, Hythe, Kent, CT21 5JU, United Kingdom

* Correspondence: Dr Yuri G. Anissimov,

Telephone: (617) 55528496; Fax: (617) 55528065; E-mail: Y.Anissimov@Griffith.edu.au

Running Head: Modelling skin penetration

\begin{abstract}
The Laplace transform is a convenient mathematical tool for solving ordinary and partial differential equations. The application of this technique to problems arising in drug penetration through the skin is reviewed in this paper.
\end{abstract}

Keywords: Laplace transform, mathematical modelling, percutaneous drug delivery

\section{Introduction}

It is well recognised [1] that application of the Laplace transform technique to problems on modelling transdermal drug delivery was pioneered by Jonathan Hadgraft (e.g. see [2-4]). In the authors' view, this awareness of mathematics and readiness in applying it to the selected field of study is rather rare for pharmaceutical scientists, especially those scholars whose first degree is in neither physics nor mathematics. As well as using the Laplace transform technique Jon later went on to champion the use of 'compartmental' modelling of transdermal delivery (e.g. see [5-7]), using software such as Stella ${ }^{\circledR}$ to improve the accessibility of mathematical approaches to predicting 
percutaneous absorption. Alongside his contribution to the use of mathematics in the drug delivery field Jon has utilised his knowledge of physical chemistry in several key papers that address the mechanistics of skin penetration enhancement [8-12] and he remains active in the many debates that still rage in this arena. Professor Hadgraft's mathematical knowledge is an example for present and future pharmaceutical scientists as is his ability to often make his point with grace and humour. One such occasion occurred at a Gordon Conference debate about the utility of electron microscopy in the study of skin structure. Jon and one of the authors of this article were up until the small hours of the morning constructing a presentation that would bring the debate to a conclusion, hopefully suggesting that 'the artefact' was a real problem in the interpretation of electron micrographs of skin. The creation of this presentation was aided, to a degree, by the consumption of some rather good Scotch and was based around challenging proponents of the technique to tell the difference between electron micrographs of the skin and various photographs of the solar system, its moons and planets. Common features of the skin were generously pointed out with increasingly large arrows on the pictures taken in space and the advocates of electron microscopy failed to tell if these were celestial objects or highly magnified images of the skin. The audience was easily won over and the debate convincingly won. There are many other examples of how Jon has used his good nature to win arguments that he actually feels quite strongly about and his rigorous approach to science, combined with a razor sharp brain and an excellent sense of humour and humility has made him one of the most valued contributors to our field over the last 40 years or so.

In this paper we have tried to present a brief overview of the Laplace transform and a detailed example of its application to a simple case followed by a review of many problems in the skin penetration literature solved by Laplace transform. It is hoped that this format could be useful and understandable (at least in part) for scholars with a non-mathematical background.

\section{Laplace transform}

The Laplace transform technique was developed at the end of the $18^{\text {th }}$ century by French mathematician and astronomer Pierre-Simon Laplace. He later used his transform to find solutions for the diffusion equation, which is at the centre of modelling drug penetration and transport through the skin. In the notation relevant to the skin transport literature, Laplace transform of the concentration $C$ at the position $x$ in the skin and time $t, C(x, t)$, is defined as: 


$$
\hat{C}(x, s)=\mathcal{L}\{C(x, t)\}=\int_{0}^{\infty} C(x, t) e^{-s t} d t
$$

where $\mathcal{L}$ is the Laplace operator, $s$ is the Laplace variable and the hat over function ( ${ }^{`}$ ) denotes the Laplace transform. If the Laplace transform of a function is known it can be inverted to time domain:

$$
C(x, t)=\mathcal{L}^{-1}\{\hat{C}(x, s)\}=\int_{\sigma-i \infty}^{\sigma+i \infty} \hat{C}(x, s) e^{s t} d s
$$

where $\mathcal{L}^{-1}$ is the inverse Laplace operator. Inverting to time domain using equation (2) is not trivial and requires the knowledge of the theory of functions of a complex variable, but often tables of Laplace transform (e.g.[13]) could help the inversion process.

The essential property of the Laplace transform in the context of solving differential equations is that derivatives with respect to time are converted to multiplication by the Laplace variable:

$$
\mathcal{L}\left\{\frac{\partial}{\partial t} C(x, t)\right\}=s \hat{C}(x, s)-C(x, 0)
$$

and thus reduces the partial differential diffusion equation to ordinary differential equation that is much easier to solve.

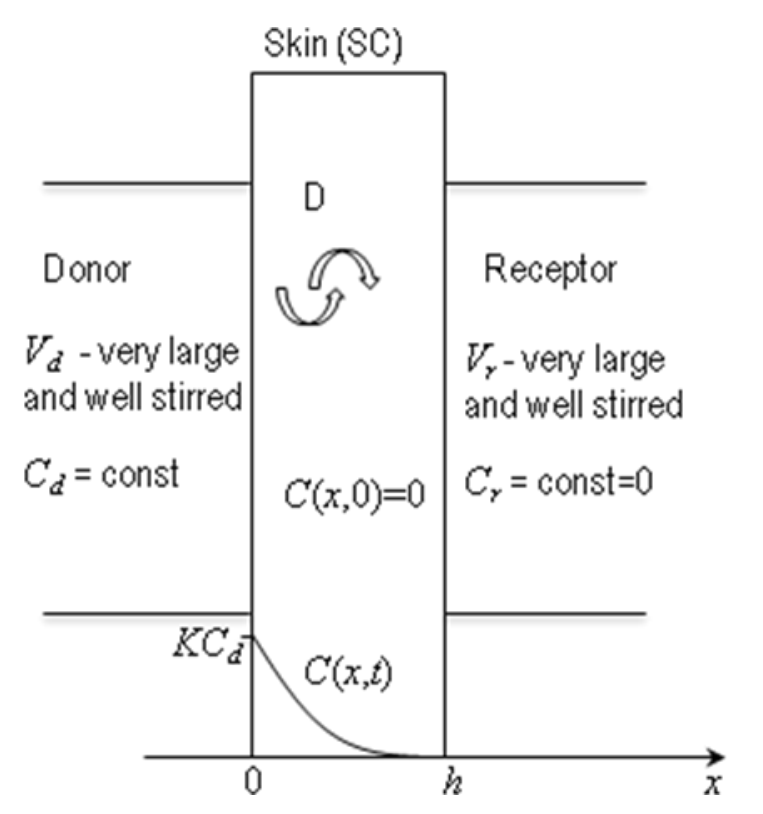

Fig 1. Schematics of the simple case of drug penetration through skin.
Application of the Laplace transform is best understood in the context of a simple example: let us consider an experiment where a relatively large volume donor solution is applied to the external surface of the skin at $t=0$ and there is no drug in the skin before the application (also see Fig 1). For simplicity we will assume that the skin can be represented as a homogeneous membrane of thickness $h$. Further, the clearance of a solute due to blood flow (or receptor stirring in the case of the in vitro diffusion cell experiment) is fast, so that the sink condition is maintained at the internal surface of the skin. The problem is to find concentration in the skin and flux through the skin. 
Transport of solutes in the homogeneous membrane is governed by the diffusion equation: [14]

$$
\frac{\partial C}{\partial t}=D \frac{\partial^{2} C}{\partial x^{2}}
$$

While the diffusion equation (4) remains the same for many problems of modelling skin solute transport, the specifics of a given problem are usually expressed in boundary conditions. In the simple case considered in this example, a large volume and well stirred donor solution provides constant concentration in the receptor phase, therefore:

$$
C(0, t)=K C_{d}
$$

where $C_{d}$ is the constant donor concentration and $K$ is the partitioning coefficient between the skin and the donor phase. The boundary condition (5) represents the fact that for any time $(t>0)$ concentration in the superficial layer of the skin (at $x=0$ ) is proportionate to the concentration in the adjacent donor solution. The coefficient of the proportionality $(K)$ in equation (5) reflects different physicochemical properties of the donor phase and the skin, e.g. if solute has greater affinity for the skin compared to the donor phase then $K>1$. The assumption of a large volume in the receptor is necessary to provide virtually constant donor concentration, that is even though solute diffuses into and through the skin and thus some solute is being lost from the donor, the loss is much smaller than the amount present in the donor ( $=$ Dose $=C_{d} V_{d}$, where $V_{d}$ is the volume of the donor) and can be neglected. Practically, if the loss is less than about $10 \%$, the assumption of constant donor concentration is justified. The assumption of a well stirred donor guarantees constant concentration throughout entire donor phase. Theoretically, if no stirring is present, the donor volume adjacent to the skin may get depleted, even when only small amount of solute diffuses into and through the skin. Practically, the diffusion through skin is so slow that even thermal convection in the donor phase may provide enough stirring to maintain a well stirred donor. Stirring the donor may become important when the partitioning coefficient is very large $(K \gg 1)$.

The second boundary condition describes the sink condition at the internal surface of the skin $(x=$ h):

$$
C(h, t)=0
$$

Essentially, equation (6) is analogues to (5), but concentration in the receptor $\left(C_{r}\right)$ in vitro or arterial blood in vivo is assumed to be zero. For the in vitro case stirring the receptor is often more important than stirring the donor, as stirring the receptor not only serves to provide the sink condition, but also provides representative sampling of the receptor phase (e.g. 10\% 
inhomogeneities of the concentration in the receptor will lead to $10 \%$ error in determination of the flux through the skin).

Second order partial differential equation (4) requires an initial condition, which is readily supplied by the assumption that there is no drug in the skin at $t=0$ :

$$
C(x, 0)=0
$$

Taking the Laplace transform of the diffusion equation (4) using equation (3) and then the initial condition (7) yields:

$$
s \hat{C}(x, s)=D \frac{d^{2} \hat{C}(x, s)}{d x^{2}}
$$

where we used the fact that $\mathcal{L}\left\{\frac{\partial^{2}}{\partial x^{2}} C(x, t)\right\}=\frac{\partial^{2}}{\partial x^{2}} \mathcal{L}\{C(x, t)\}=\frac{d^{2}}{d x^{2}} \hat{C}(x, s)$ (as there is no derivative with any other variable in (8), partial derivative with $x$ can be replaced with full derivative). The Laplace transform of the boundary conditions (5) and (6) yields:

$$
\begin{gathered}
\hat{C}(0, s)=\frac{K C_{d}}{s} \\
\hat{C}(h, s)=0
\end{gathered}
$$

Equation (8) is a simple ordinary second order differential equation, which is much easier to solve than the original partial differential equation (4), and has a well known solution:

$$
\hat{C}(x, s)=A \sinh \left(x \sqrt{\frac{s}{D}}\right)+B \cosh \left(x \sqrt{\frac{s}{D}}\right)
$$

where $A$ and $B$ are constants to be determined from using boundary conditions (9) and (10). Given that $\sinh (x)=(\exp (x)-\exp (-x)) / 2$ and $\cosh (x)=(\exp (x)+\exp (-x)) / 2$ equation (11) can also be expressed using exponentials, but for a problem involving finite thickness membrane (or few membranes) the notation used in equation (11) leads to simpler mathematical expressions. It is convenient to "repackage" expression $x \sqrt{s / D}$ in equation (11) to the term that has a more obvious dimension: $\frac{x}{h} \sqrt{s t_{d}}$, where $t_{d}=h^{2} / D$ is the characteristic time of diffusion (and since the dimension of the Laplace variable $s$ is $1 /$ time, the term is obviously dimensionless). Substitution of 
equation (11) to boundary condition (9) yields $B=K C_{d} / s$, and then the use of boundary condition (10) determines the constant $A$, so that:

$$
\hat{C}(x, s)=\frac{K C_{d}}{s}\left[\cosh \left(\frac{x}{h} \sqrt{s t_{d}}\right)-\frac{\cosh \left(\sqrt{s t_{d}}\right)}{\sinh \left(\sqrt{s t_{d}}\right)} \sinh \left(\frac{x}{h} \sqrt{s t_{d}}\right)\right]
$$

Using Hyperbolic functions identities this equation can be expressed as: [15]

$$
\hat{C}(x, s)=\frac{K C_{d}}{s} \frac{\sinh \left(\sqrt{s t_{d}}(1-x / h)\right)}{\sinh \left(\sqrt{s t_{d}}\right)}
$$

Equation (13) can be inverted numerically to time domain (see next section) and used directly to fit experimental data (such as skin tape stripping data, e.g. [16]), but some information and insight can be gained directly from the Laplace expression (13). This can be done using the following relationship:

$$
\lim _{t \rightarrow \infty} f(t)=\lim _{s \rightarrow 0} s \hat{f}(s)
$$

It is important to note that equation (14) is only valid if $\lim _{t \rightarrow \infty} f(t)$ exists (and is therefore finite). For example at long times ( $t \gg t_{d}$, but practically for $\left.t>t_{d} / 2\right)$ the steady state concentration profile $\left(C_{s s}(x)\right)$ can be easily found:

$$
C_{s s}(x)=\lim _{t \rightarrow \infty} C(x, t)=\lim _{s \rightarrow 0} s \hat{C}(x, s)=K C_{d}\left(1-\frac{x}{h}\right)
$$

At very short times $\left(t \ll t_{d}\right)$ in equation (13) large values of $s$ (so that $s t_{d} \gg 1$ ) must be considered, and as $\sinh (x) \approx \exp (x) / 2$ for $x \gg 1$, equation (13) simplifies to:

$$
\hat{C}(x, s) \approx \frac{K C_{d}}{s} \exp \left(-\frac{x}{h} \sqrt{s t_{d}}\right)
$$

The inverse Laplace transform for equation (16) can be easily found using the table of Laplace transform (e.g. [13]):

$$
C(x, t) \approx \mathcal{L}^{-1}\left\{\frac{K C_{d}}{s} \exp \left(-\frac{x}{h} \sqrt{s t_{d}}\right)\right\}=K C_{d} \operatorname{erfc}\left(\frac{x}{2 h} \sqrt{\frac{t_{d}}{t}}\right)
$$

where $\operatorname{erfc}(x)$ is the complementary error function (which can be calculated for example in Excel).

The flux of solute from the skin to the receptor $(J)$ can be determined for the given concentration: 


$$
J(t)=-\left.D \frac{\partial C(x, t)}{\partial x}\right|_{x=h}
$$

in the Laplace domain this equation is:

$$
\hat{J}(s)=-\left.D \frac{d \hat{C}(x, s)}{d x}\right|_{x=h}
$$

Using $\hat{C}(x, s)$ from equation (13) in (19) yields for flux:

$$
\hat{J}(s)=\frac{K D C_{d}}{h} \frac{\sqrt{s t_{d}}}{s \sinh \left(\sqrt{s t_{d}}\right)}
$$

Using equation (14) with equation (20) the steady state flux ( $J_{s s}$ ) can be easily found:

$$
J_{s s}=\lim _{t \rightarrow \infty} J(t)=\lim _{s \rightarrow 0} s \hat{J}(s)=\frac{K D}{h} C_{d}=k_{p} C_{d}
$$

where $k_{p}$ is the permeability coefficient of the skin. It is often preferable to express equation (20) and (21) in terms of the maximum flux of a solute through skin:

$$
J_{s s}=\frac{K D}{h} C_{d}=k_{p} C_{d}=J_{\max } \frac{C_{d}}{S_{d}}
$$

where $J_{\max }$ is the maximum flux of a solute through skin, realised when maximum possible concentration (= solubility in the donor, $S_{d}$ ) for a given donor phase is used. The advantage of equation (22) is that $J_{\max }$ unlike $k_{p}$ is a donor phase independent parameter, providing that the donor phase does not change the transport properties of the skin. [17]

In skin diffusion experiments the data is often expressed as the total amount $(Q)$ of solute penetrated into the receptor phase (or systemically absorbed, for the in vivo case). The total amount can be readily found from the flux:

$$
Q(t)=\int_{0}^{t} A J(\tau) d \tau
$$

where $A$ is the area of the skin in contact with the donor and receptor phases. Since integration is inverse operation to differentiation (and derivative corresponds to multiplying by s, see equation (3) ) it is intuitively clear that the Laplace transform of an integral corresponds to multiplication by $s^{-1}=1 / s$, and the Laplace transform of (23) yields:

$$
\hat{Q}(s)=\frac{A \hat{J}(s)}{s}
$$

Together with equation (20) this gives: 


$$
\hat{Q}(s)=\frac{K D A C_{d}}{h} \frac{\sqrt{s t_{d}}}{s^{2} \sinh \left(\sqrt{s t_{d}}\right)}
$$

At long times (practically $t>t_{d} / 2$ )

$$
Q(t) \approx A J_{s s}(t-\operatorname{lag})
$$

where lag is the lag time which can be expressed using $\hat{Q}(s):[17,18]$

$$
\operatorname{lag}=-\lim _{s \rightarrow 0} \frac{d}{d s} \ln \left(s^{2} \hat{Q}(s)\right)=\frac{t_{d}}{6}
$$

\section{Numerical inverse Laplace transform}

The usefulness of Laplace domain solutions have been enhanced by implementation of numerical inverse Laplace transform algorithms in readily available nonlinear regression programs such as MULTI FILT, MINIM and SCIENTIST. These software packages permit fitting experimental data points directly using Laplace domain solutions, avoiding mathematical complexities associated with obtaining infinite series time domain solutions which, in more advanced models, also require solving transcendental equations.

While using the software packages is sufficient in most cases of experimental data analysis in modelling skin penetration, there are problems that require a more flexible approach. For example, the abovementioned software packages do not allow batch processing of data, which is inconvenient when fitting large massive of data. One of the authors encountered an insurmountable problem trying to use SCIENTIST with the Laplace solution involving Bessel functions [19]. To address these problems programming languages, such as Fortran or Python can be used. For reader's convenience Python program for numerical inverse Laplace transform is presented in the Appendix.

\section{Various problems in skin penetration literature solved by Laplace transform}

Table 1 lists common models of skin penetration with schematics, brief description and Laplace solution equations for the flux into the receptor phase $(\hat{J}(s))$, amount absorbed into the receptor phase $(\hat{Q}(s))$, and concentration in the skin (or Stratum Corneum, SC, $\hat{C}(x, s)$ ) when available. Equations are normally given for simpler models with fewer parameters and, when possible, have been converted to the notations consistent with this work. 
Table 1

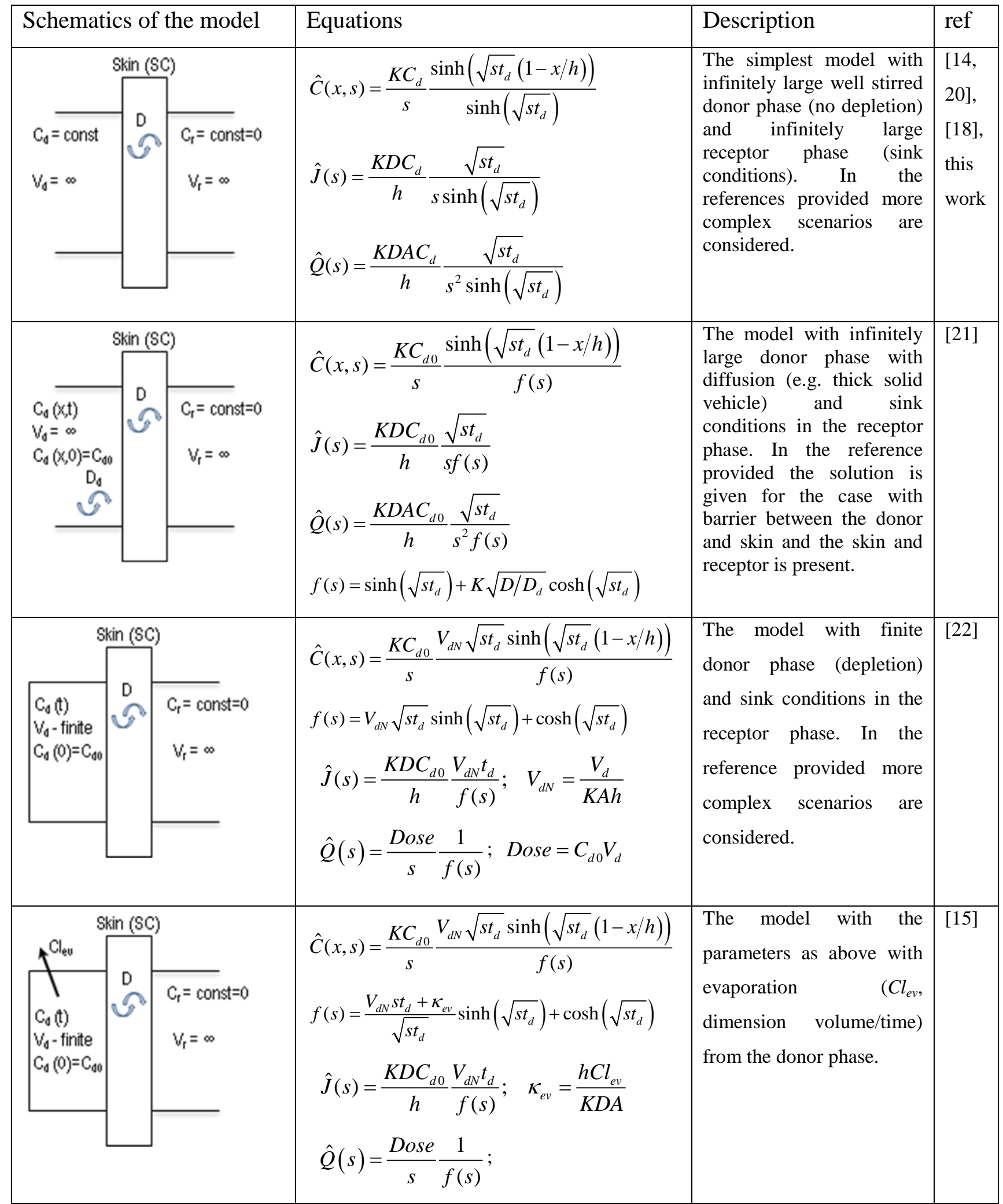




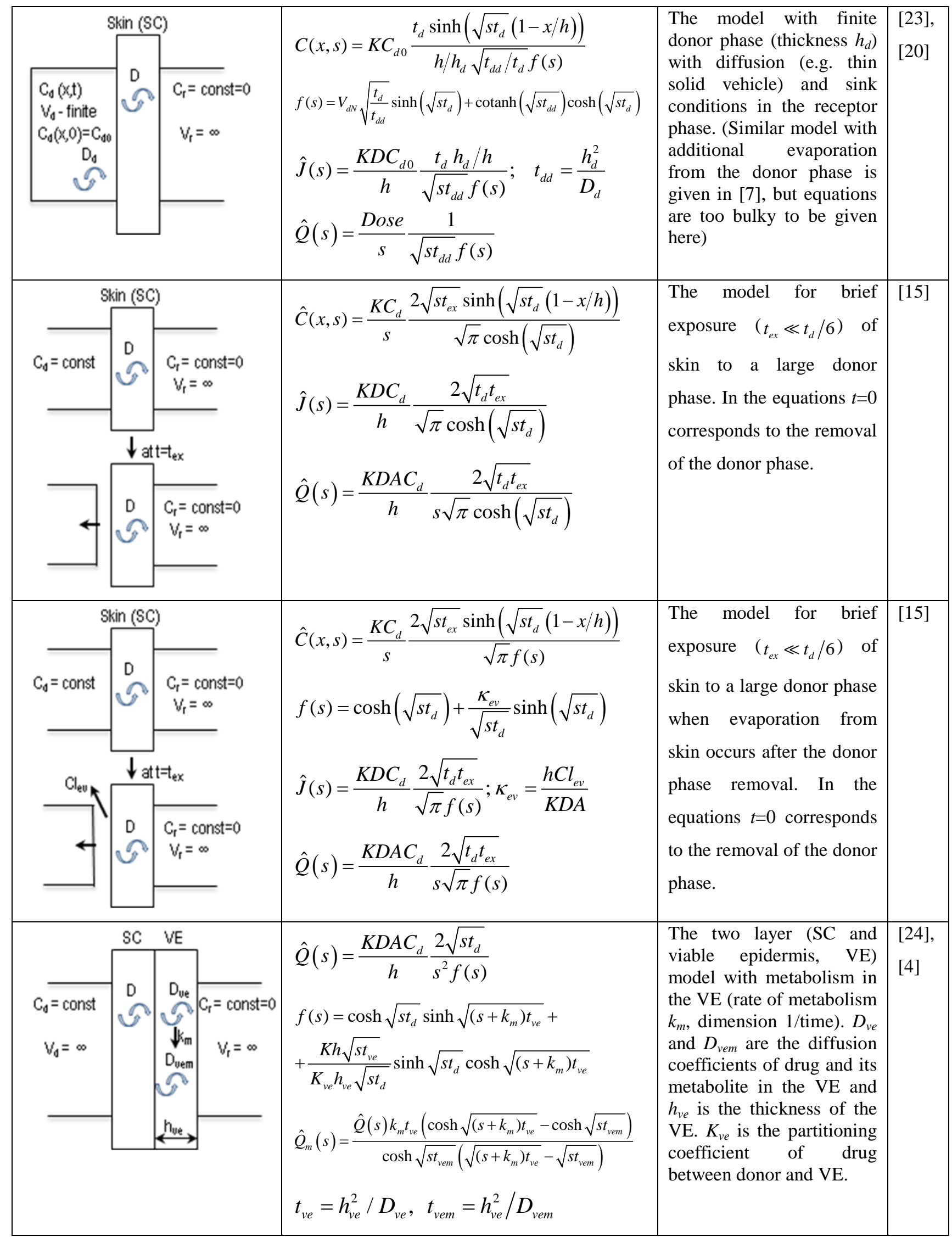




\begin{tabular}{|c|c|c|c|c|c|}
\hline \multirow[b]{2}{*}{$\begin{array}{l}C_{4}=\text { const } \\
V_{4}=\infty\end{array}$} & \multirow{2}{*}{ 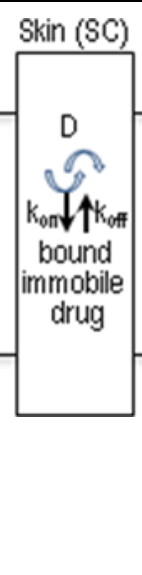 } & \multirow{2}{*}{$\begin{array}{l}C_{\mathrm{r}}=\text { const }=0 \\
\mathrm{~V}_{\mathrm{r}}=\infty\end{array}$} & \multirow{2}{*}{$\begin{array}{l}\hat{C}(x, s)=\frac{f_{u} K C_{d}}{s} \frac{g(s) \sinh \left(\sqrt{g(s) t_{d}}(1-x / h)\right)}{s \sinh \left(\sqrt{g(s) t_{d}}\right)} \\
g(s)=s+\frac{s k_{\text {on }}}{s+k_{\text {off }}}, \quad f_{u}=\left(1+\frac{k_{\text {on }}}{k_{\text {off }}}\right)^{-1} \\
\hat{J}(s)=\frac{K D C_{d}}{h} \frac{\sqrt{g(s) t_{d}}}{s \sinh \left(\sqrt{g(s) t_{d}}\right)} \\
\hat{Q}(s)=\frac{K D A C_{d}}{h} \frac{\sqrt{g(s) t_{d}}}{s^{2} \sinh \sqrt{g(s) t_{d}}}\end{array}$} & \multirow{2}{*}{$\begin{array}{l}\text { The model when solute } \\
\text { undergoes rate limiting } \\
\text { slow binding in the SC for } \\
\text { example due to } \\
\text { partitioning into } \\
\text { corneocytes. Here } k_{\text {on }} \text { and } \\
k_{\text {off }} \text { are binding and } \\
\text { unbinding rate constants } \\
\text { (dimension } 1 / \text { time). }\end{array}$} & \multirow{2}{*}{$\begin{array}{l}{[25]} \\
{[17]}\end{array}$} \\
\hline & & & & & \\
\hline $\begin{array}{l}C_{4}=\text { const } \\
V_{4}=\infty\end{array}$ & $\mathrm{SC}$ & $\mathrm{C}_{4}=$ const & $\begin{array}{l}\hat{C}(x, s)=\frac{K C_{d}}{s} \frac{\sinh \left(\sqrt{s t_{d}}(1-x / h)\right)+\sinh \left(\sqrt{s t_{d}} x / h\right)}{\sinh \left(\sqrt{s t_{d}}\right)} \\
\hat{J}(s)=\frac{K D C_{d}}{h} \frac{2 \sqrt{s t_{d}} \tanh \left(\sqrt{s t_{d}} / 2\right)}{s} \\
\hat{Q}(s)=\frac{K D A C_{d}}{h} \frac{2 \sqrt{s t_{d}} \tanh \left(\sqrt{s t_{d}} / 2\right)}{s^{2}}\end{array}$ & $\begin{array}{l}\text { The model for sorption. } \\
\text { SC originally devoid of } \\
\text { solute is immersed into the } \\
\text { donor solution. Flux and } \\
\text { amount of drug absorbed } \\
\text { into the SC is given here. }\end{array}$ & [17] \\
\hline$V_{r}=\infty$ & SC & $V_{T}=\infty$ & $\begin{array}{l}\hat{C}(x, s)=\frac{K C_{d}}{s}\left[1-\frac{\sinh \left(\sqrt{s t_{d}}(1-x / h)\right)+\sinh \left(\sqrt{s t_{d}} x / h\right)}{\sinh \left(\sqrt{s t_{d}}\right)}\right] \\
\hat{J}(s)=\frac{K D C_{d}}{h} \frac{2 \sqrt{s t_{d}} \tanh \left(\sqrt{s t_{d}} / 2\right)}{s} \\
\hat{Q}(s)=\frac{K D A C_{d}}{h} \frac{2 \sqrt{s t_{d}} \tanh \left(\sqrt{s t_{d}} / 2\right)}{s^{2}}\end{array}$ & $\begin{array}{l}\text { The model for desorption. } \\
\text { SC that has concentration } \\
C(x, 0)=K C_{d} \text { at } t=0 \text { is } \\
\text { immersed into the receptor } \\
\text { solution. }\end{array}$ & $\begin{array}{l}\text { [17], } \\
{[19]}\end{array}$ \\
\hline$V_{t}=\infty$ & $\begin{array}{c}\underbrace{D} \\
k_{\text {ond }} \downarrow \uparrow k_{\text {off }} \\
\text { bound } \\
\text { immobile } \\
\text { drug }\end{array}$ & $\begin{array}{l}C_{t}=0 \\
V_{t}=\infty\end{array}$ & $\begin{array}{l}\hat{C}(x, s)=\frac{K C_{d}}{s} f(s) \times \\
{\left[1-\frac{\sinh \left(\sqrt{g(s) t_{d}}(1-x / h)\right)+\sinh \left(\sqrt{g(s) t_{d}} x / h\right)}{\sinh \left(\sqrt{g(s) t_{d}}\right)}\right]} \\
f(s)=\left(f_{u}+\frac{k_{\text {off }}\left(1-f_{u}\right)}{s+k_{\text {off }}}\right) \\
g(s)=s+\frac{s k_{\text {on }}}{s+k_{\text {off }}}, \quad f_{u}=\left(1+\frac{k_{\text {on }}}{k_{\text {off }}}\right)^{-1} \\
\hat{J}(s)=K h C_{d} \frac{2 f(s) \tanh \left(\sqrt{g(s) t_{d}} / 2\right)}{\sqrt{g(s) t_{d}}} \\
\hat{Q}(s)=\frac{K A h C_{d}}{s} \frac{2 f(s) \tanh \left(\sqrt{g(s) t_{d}} / 2\right)}{\sqrt{g(s) t_{d}}}\end{array}$ & $\begin{array}{l}\text { The model for desorption } \\
\text { when slow binding occurs } \\
\text { in the SC. SC that has } \\
\text { concentration } \\
C(x, 0)=K C_{d} \text { at } t=0 \text { is } \\
\text { immersed into the receptor } \\
\text { solution. }\end{array}$ & $\begin{array}{l}{[17],} \\
{[25]}\end{array}$ \\
\hline
\end{tabular}




\section{Conclusion}

We hope in this work we have demonstrated that the Laplace transform is a powerful mathematical tool for solving the diffusion equation and analysing solutions for solute transport in skin. It is important to recognise that this tool has its limitations. Most notably, to be solvable by the Laplace transform partial differential equations have to have coefficients that are independent of time (e.g. constants or functions of $x$ only). This excludes an important class of problems in skin transport that involve diffusion coefficient that is changing in time. For example changing diffusion coefficient can result from drying of skin due to exposure to an environment. Another limitation is that the Laplace transform only works for linear differential equations, and therefore will not work for a model with diffusion coefficient that is dependent on concentration. This scenario is likely for example in a case of diffusion or co-diffusion of a penetration enhancer. In the cases that the Laplace transform could not be used, numerical solution of the diffusion equation has to be undertaken and Laplace solutions can be used to finetune the numerical solution. An example of the numerical approach, that represents skin as an array of compartments, is discussed in $[15,17,26]$ and gives required flexibility to address difficult skin penetration scenarios.

\section{Appendix}

Presented below is Python program for inverting function described by equation (25) (adapted from http://code.activestate.com/recipes/576938-numerical-inversion-of-the-laplace-transform-with/history/1/, see also [27]). The algorithm is based on using inverse Laplace integral (equation (2)) directly, but modifying the integration paths from the straight line in equation (2) to a properly selected curve to speed the convergence of the integral.

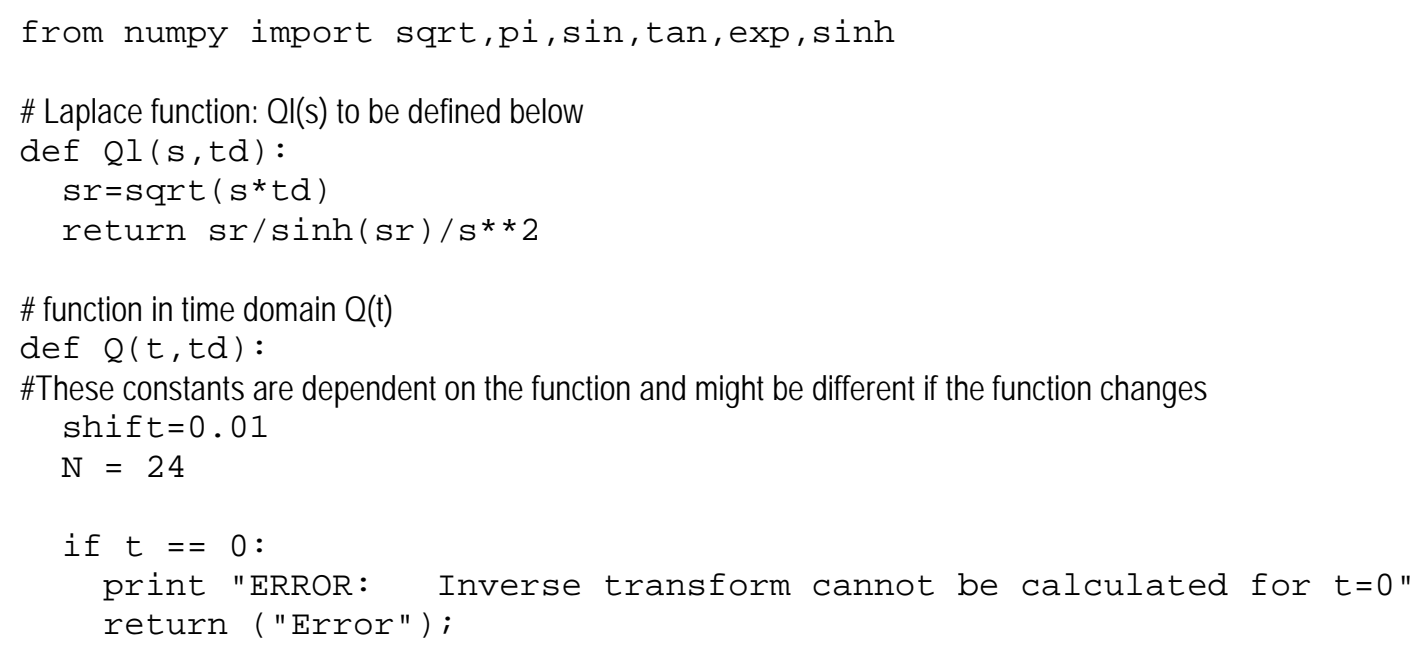




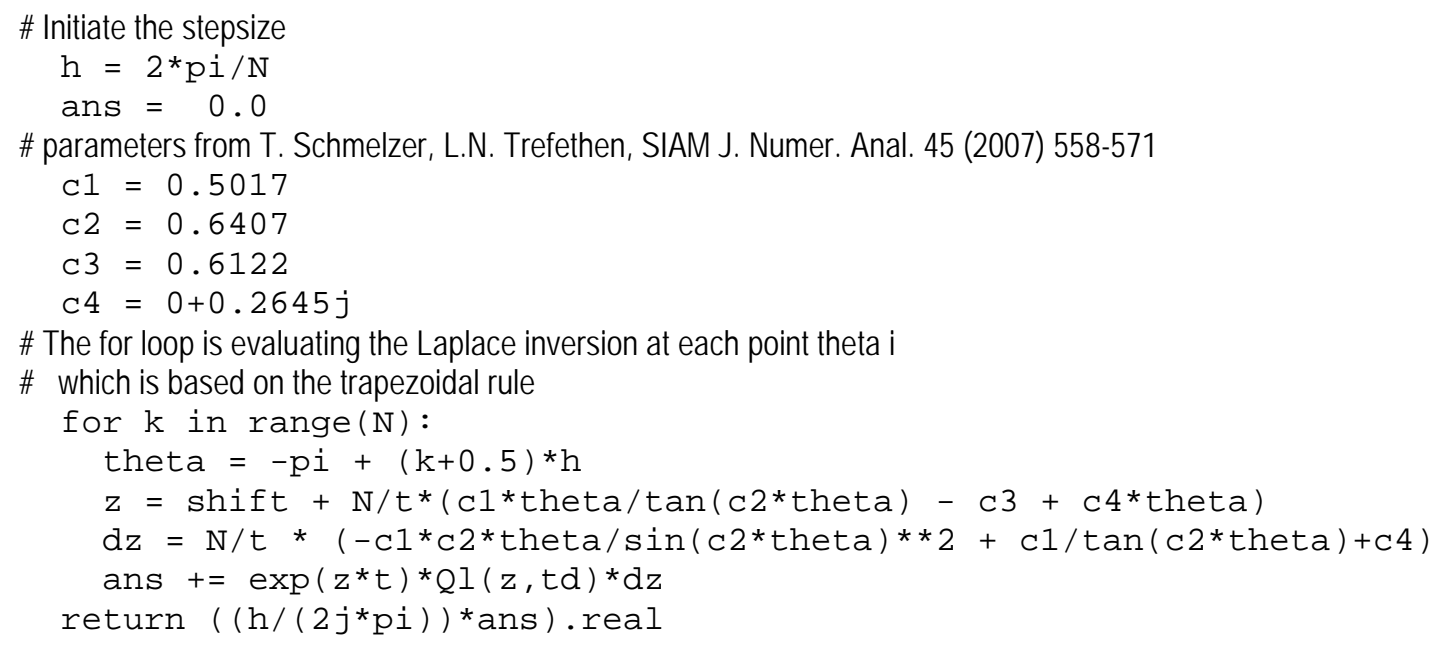

\section{References}

[1] S. Mitragotri, Y.G. Anissimov, A.L. Bunge, H.F. Frasch, R.H. Guy, J. Hadgraft, G.B. Kasting, M.E. Lane, M.S. Roberts, Mathematical models of skin permeability: An overview, Int. J. Pharm., 418 (2011) 115-129.

[2] J. Hadgraft, Calculations of drug release from controlled release devices. The slab, Int. J. Pharm., 2 (1979) 177-194.

[3] J. Hadgraft, The epidermal reservoir: a theoretical approach., Int. J. Pharm., 2 (1979) 265-274.

[4] J. Hadgraft, Theoretical aspects of metabolism in the epidermis., Int. J. Pharm., 4 (1980) 229239.

[5] R.H. Guy, J. Hadgraft, Prediction of drug disposition kinetics in skin and plasma following topical administration, J. Pharm. Sci., 73 (1984) 883-887.

[6] R.H. Guy, J. Hadgraft, Pharmacokinetics of percutaneous absorption with concurrent metabolism, Int. J. Pharm., 20 (1984) 43-51.

[7] R.H. Guy, J. Hadgraft, Percutaneous absorption kinetics of topically applied agents liable to surface loss, Journal of the Society of Cosmetic Chemists, 35 (1984) 103-113.

[8] R.H. Guy, J. Hadgraft, Physicochemical aspects of percutaneous penetration and its enhancement, Pharm. Res., 5 (1988) 753-758.

[9] J. Hadgraft, Passive enhancement strategies in topical and transdermal drug delivery, Int. J. Pharm., 184 (1999) 1-6.

[10] M. Iervolino, B. Cappello, S.L. Raghavan, J. Hadgraft, Penetration enhancement of ibuprofen from supersaturated solutions through human skin, Int. J. Pharm., 212 (2001) 131-141.

[11] M. Iervolino, S.L. Raghavan, J. Hadgraft, Membrane penetration enhancement of ibuprofen using supersaturation, Int. J. Pharm., 198 (2000) 229-238.

[12] L. Trottet, C. Merly, M. Mirza, J. Hadgraft, A.F. Davis, Effect of finite doses of propylene glycol on enhancement of in vitro percutaneous permeation of loperamide hydrochloride, Int. J. Pharm., 274 (2004) 213-219.

[13] M. Abramowitz, I.A. Stegun, Handbook of mathematical functions with formulas, graphs, and mathematical tables, Dover, New York, NY, 1965.

[14] J. Crank, The mathematics of diffusion, 2 ed., Clarendon Press, Oxford, 1975.

[15] Y.G. Anissimov, Mathematical Models for Different Exposure Conditions, in: M.S. Roberts, K.A. Walters (Eds.) Dermal Absorption and Toxicity Assessment Informa Healthcare, New York, 2008, pp. 271-286. 
[16] M.B. Reddy, A.L. Stinchcomb, R.H. Guy, A.L. Bunge, Determining dermal absorption parameters in vivo from tape strip data, Pharm. Res., 19 (2002) 292-298.

[17] Y.G. Anissimov, O.G. Jepps, Y. Dancik, M.S. Roberts, Mathematical and pharmacokinetic modelling of epidermal and dermal transport processes, Adv Drug Deliv Rev, Available online 7 May 2012 (2012).

[18] Y.G. Anissimov, M.S. Roberts, Diffusion modeling of percutaneous absorption kinetics: 1. Effects of flow rate, receptor sampling rate and viable epidermal resistance for a constant donor concentration, J. Pharm. Sci., 88 (1999) 1201-1209.

[19] Y.G. Anissimov, M.S. Roberts, Diffusion modeling of percutaneous absorption kinetics: 3. Variable diffusion and partition coefficients, consequences for stratum corneum depth profiles and desorption kinetics, J. Pharm. Sci., 93 (2004) 470-487.

[20] M.S. Roberts, Y.G. Anissimov, Mathematical models in percutaneous absorption, in: R.L. Bronaugh, H.I. Maibach (Eds.) Percutaneous Absorption Drugs -- Cosmetics -- Mechanisms -Methodology, Marcel Dekker, New York, 2005, pp. 1--44.

[21] W.J. Albery, J. Hadgraft, Percutaneous absorption: theoretical description., J. Pharm. Pharmacol., 31 (1979) 129-139.

[22] Y.G. Anissimov, M.S. Roberts, Diffusion modeling of percutaneous absorption kinetics: 2. Finite vehicle volume and solvent deposited solids, J. Pharm. Sci., 90 (2001) 504-520.

[23] R.H. Guy, J. Hadgraft, A theoretical description relating skin penetration to the thickness of the applied medicament, Int. J. Pharm., 6 (1980) 321-332.

[24] N. Seko, H. Bando, C.W. Lim, F. Yamashita, M. Hashida, Theoretical analysis of the effect of cutaneous metabolism on skin permeation of parabens based on a two-layer skin diffusion/metabolism model, Biol. Pharm. Bull., 22 (1999) 281-287.

[25] Y.G. Anissimov, M.S. Roberts, Diffusion modeling of percutaneous absorption kinetics: 4. Effects of a Slow Equilibration Process Within Stratum Corneum on Absorption and Desorption Kinetics., J. Pharm. Sci., 98 (2009) 772-781.

[26] J. Zatz, Simulation studies of skin permeation, J. Soc. Cosm. Chem., 43 (1992) 37-48.

[27] L.N. Trefethen, J.A.C. Weideman, T. Schmelzer, Talbot quadratures and rational approximations, Bit, 46 (2006) 653-670. 